\title{
CONSENSUS GAME ACCEPTORS AND ITERATED TRANSDUCTIONS
}

\author{
DIETMAR BERWANGER AND MARIE VAN DEN BOGAARD \\ LSV, CNRS and Université Paris-Saclay, France
}

\begin{abstract}
We study a game for recognising formal languages, in which two players with imperfect information need to coordinate on a common decision, given private input words correlated by a finite graph. The players have a joint objective to avoid an inadmissible decision, in spite of the uncertainty induced by the input.

We show that the acceptor model based on consensus games characterises context-sensitive languages. Further, we describe the expressiveness of these games in terms of iterated synchronous transductions and identify a subclass that characterises context-free languages.
\end{abstract}

\section{INTRODUCTION}

The idea of viewing computation as an interactive process has been at the origin of many enlightening developments over the past three decades. With the concept of alternation, introduced around 1980 by Chandra and Stockmeyer, and independently by Kozen [6], computation steps are attributed to conflicting players seeking to reach or avoid certain outcome states. This approach relies on determined games with perfect information, and it led to important and elegant results, particularly in automata theory. Around the same time, Peterson and Reif [19] initiated a study on computation via games with imperfect information, involving teams of players. This setting turned out to be highly expressive, but also overwhelmingly difficult to comprehend. (See [2, 11, for more recent accounts.)

In this paper, we propose a game model of a language acceptor based on coordination games between two players with imperfect information. Compared to the model of Reif and Peterson, our setting is utterly simple: the games are played on a finite graph, plays are of finite duration, they involve only one yes-or-no decision, and the players have no means to communicate. Moreover, they are bound to take their decisions in consensus. Given an input word that may yield different observations to each of the players, they have to settle simultaneously and independently on a common decision, otherwise they lose.

E-mail address: dwb@lsv.fr (Dietmar Berwanger, corresponding author). 
Consensus game acceptors arise as a particular case of coordination games with perfect recall, also described as multiplayer concurrent games or synchronous distributed games with incomplete information in the computerscience literature. Our motivation for studying the acceptor model is to obtain lower bounds on the complexity of basic computational problems on coordination games with imperfect information, specifically (1) solvability: whether a winning strategy exists for a given game, and (2) implementability: which computational resources are needed to implement a winning strategy, if any exists.

Without the restrictions to consensus and to a single decision per play, the solvability problem for coordination games with safety winning conditions is known to be undecidable [19, 20]. Furthermore, Janin [12] points out that there exist two-player safety games that admit a winning strategy but none that can be implemented by a Turing machine.

Our first result establishes a correspondence between context-sensitive languages and consensus games: We prove that, for every context-sensitive language $L$, there exists a solvable consensus game in which every winning strategy extends the characteristic function of $L$, and conversely, that every solvable consensus game admits a winning strategy characterised by a context-sensitive language.

As a second result, we characterise winning strategies for consensus games in terms of iterated transductions of the (synchronous rational) relation between the observations of players. This allows us to identify a subclass of games that corresponds to context-free languages. Although it is still undecidable whether a game of the class admits a winning strategy, we can effectively construct optimal strategies implemented by push-down automata.

The results provide insight on the inherent complexity of coordination in games with imperfect information. With regard to the basic problem of agreement on a simultaneous action, they substantiate the assertion that "optimality requires computing common knowledge" put forward by Dwork and Moses in their analysis of Byzantine agreement in distributed systems $[9$. Indeed, the constraints induced by our acceptor model can be reproduced in virtually any kind of games with imperfect information and plays of unbounded length, with the consequence that implementing optimal strategies amounts to deciding the transitive closure of the transduction induced by the game graph.

Acknowledgements. This work was partially supported by CASSTING Project of the European Union Seventh Framework Programme. The current paper extends a preliminary report [5] presented at the Conference on Developments in Language Theory (DLT 2015).

\section{Preliminaries}

For standard background on formal language theory, in particular contextsensitive languages, we refer to Chapters 3 and 4 of the handbook [21]. We 
use the characterisation of context-sensitive languages in terms of nondeterministic linear-bounded automata given by Kuroda [13], and the following well-known results from the same article: (1) For a fixed context-sensitive language $L$ over an alphabet $\Sigma$, the problem whether a given word $w \in \Sigma^{*}$ belongs to $L$ is PSPACE-hard. (2) The problem of determining whether a given context-sensitive language represented by a linear-bounded automaton contains any non-empty word is undecidable.

2.1. Consensus game acceptors. Consensus acceptors are games between two cooperating players 1 and 2, and a passive agent called Input. Given a finite observation alphabet $\Gamma$ common to both players, a consensus game acceptor $G=\left(V, E,\left(\beta^{1}, \beta^{2}\right), v_{0}, \Omega\right)$ is described by a finite set $V$ of states, a transition relation $E \subseteq V \times V$, and a pair of observation functions $\beta^{i}: V \rightarrow \Gamma$ that label every state with an observation, for each player $i=1,2$. There is a distinguished initial state $v_{0} \in V$ with no incoming transition. States with no outgoing transitions are called final states; the admissibility condition $\Omega: V \rightarrow \mathcal{P}(\{0,1\})$ maps every final state $v \in V$ to a nonempty subset of admissible decisions $\Omega(v) \subseteq\{0,1\}$. The observations at the initial and the final states do not matter, we assume that they correspond to a special symbol $\# \in \Gamma$ for both players.

The game is played as follows: Input chooses a finite path $\pi=v_{0} v_{1} \ldots v_{n+1}$ in $G$ from the initial state $v_{0}$, following transitions $\left(v_{\ell}, v_{\ell+1}\right) \in E$, for all $\ell \leq n$, to a final state $v_{n+1}$. Then, each player $i$ receives a private sequence of observations $\beta^{i}(\pi):=\beta^{i}\left(v_{1}\right) \beta^{i}\left(v_{2}\right) \ldots \beta^{i}\left(v_{n}\right)$ and is asked to take a decision $a^{i} \in\{0,1\}$, independently and simultaneously. The players win if they agree on an admissible decision, that is, $a^{1}=a^{2} \in \Omega\left(v_{n+1}\right)$; otherwise they lose. Without risk of confusion we sometimes write $\Omega(\pi)$ for $\Omega\left(v_{n+1}\right)$.

We say that two plays $\pi, \pi^{\prime}$ are indistinguishable to player $i$, and write $\pi \sim^{i} \pi^{\prime}$, if $\beta^{i}(\pi)=\beta^{i}\left(\pi^{\prime}\right)$. This is an equivalence relation, and its classes, called the information sets of Player $i$, correspond to observation sequences $\beta^{i}(\pi)$. A strategy for Player $i$ is a mapping $s^{i}: V^{*} \rightarrow\{0,1\}$ from plays $\pi$ to decisions $s^{i}(\pi) \in\{0,1\}$ such that $s^{i}(\pi)=s^{i}\left(\pi^{\prime}\right)$, for any pair $\pi \sim^{i} \pi^{\prime}$ of indistinguishable plays. A joint strategy is a pair $s=\left(s^{1}, s^{2}\right)$; it is winning, if $s^{1}(\pi)=s^{2}(\pi) \in \Omega(\pi)$, for all plays $\pi$. In this case, the components $s^{1}$ and $s^{2}$ are equal, and we use the term winning strategy to refer to the joint strategy or either of its components. Finally, a game is solvable, if there exists a (joint) winning strategy.

In the terminology of distributed systems, consensus game acceptors correspond to synchronous systems with perfect recall and known initial state. They are a particular case of distributed games with safety objectives [17], coordination games with imperfect information [4], or multi-player concurrent games [1. Whenever we refer to games in the following, we mean consensus game acceptors.

2.2. Strategies and knowledge. We say that two plays $\pi$ and $\pi^{\prime}$ are connected, and write $\pi \sim^{*} \pi^{\prime}$, if there exists a sequence of plays $\pi_{1}, \ldots, \pi_{k}$ such 
that $\pi \sim^{1} \pi_{1} \sim^{2} \ldots \sim^{1} \pi_{k} \sim^{2} \pi^{\prime}$. Then, a mapping $f: V^{*} \rightarrow\{0,1\}$ from plays to decisions is a strategy that satisfies the consensus condition if, and only if, $f(\pi)=f\left(\pi^{\prime}\right)$, for all $\pi \sim^{*} \pi^{\prime}$. In terms of distributed knowledge, this means that the decisions have to be based on events that are common knowledge among the players at every play. (For an introduction to knowledge in distributed systems, see Chapters $10-11$ in the book of Fagin, Halpern, Moses, and Vardi [10].) Such a consensus strategy — or, more precisely, the pair $(f, f)$ - may still fail, due to prescribing inadmissible decisions. We say that a decision $a \in\{0,1\}$ is safe at a play $\pi$ if $a \in \Omega\left(\pi^{\prime}\right)$, for all $\pi^{\prime} \sim^{*} \pi$. Then, a consensus strategy $f$ is winning, if and only if, it prescribes a safe decision $f(\pi)$, for every play $\pi$.

It is sometimes convenient to represent a strategy for a player $i$ as a function $f^{i}: \Gamma^{*} \rightarrow\{0,1\}$. Every such function describes a valid strategy, because observation sequences identify information sets; we refer to an observationbased strategy in contrast to the state-based representation $s^{i}: V^{*} \rightarrow\{0,1\}$. Note that the components of a joint winning strategy need no longer be equal in the observation-based representation. However, once the strategy for one player is fixed, the strategy of the other player is determined by the consensus condition, so there is no risk of confusion in speaking of a winning strategy rather than a joint strategy pair.

As an example, consider the game depicted in Figure 1, with observation alphabet $\Gamma=\{a, b, \triangleleft, \triangleright, \square\}$. States $v$ at which the two players receive different observations are split, with $\beta^{1}(v)$ written in the upper part and $\beta^{2}(v)$ in the lower part; states at which the players receive the same observation carry only one symbol. The admissible decisions at final states are indicated on the outgoing arrow. Notice that upon receiving the observation sequence $a^{2} b^{2}$, for instance, the first player is constrained to choose decision 1 , due to the following sequence of indistinguishable plays that leads to a play where deciding 0 is not admissible.

$$
\left(\begin{array}{l}
a, a \\
a, \triangleleft \\
b, \triangleright \\
b, b
\end{array}\right) \sim^{2}\left(\begin{array}{c}
a, a \\
\triangleleft, \triangleleft \\
\triangleright, \triangleright \\
b, b
\end{array}\right) \sim^{1}\left(\begin{array}{c}
a, \triangleleft \\
\triangleleft, \triangleright \\
\triangleright, \triangleleft \\
b, \triangleright
\end{array}\right) \sim^{2}\left(\begin{array}{c}
\triangleleft, \triangleleft \\
\triangleright, \triangleright \\
\triangleleft, \triangleleft \\
\triangleright, \triangleright
\end{array}\right) \sim^{1}\left(\begin{array}{c}
\triangleleft, \square \\
\triangleright, \square \\
\triangleleft, \square \\
\triangleright, \square
\end{array}\right) \sim^{2}\left(\begin{array}{c}
\square, \square \\
\square, \square \\
\square, \square \\
\square, \square
\end{array}\right)
$$

In contrast, decision 0 may be safe when Player 1 receives input $a^{3} b^{2}$, for instance. Actually, the strategy $s^{1}(w)$ that prescribes 1 if, and only if, $w \in$ $\left\{a^{n} b^{n} \mid n \in \mathbb{N}\right\}$ determines a joint winning strategy. Next, we shall make the relation between games and languages more precise.

\section{Describing languages By Games}

We consider languages $L$ over a terminal alphabet $\Sigma$. The empty word $\varepsilon$ is excluded from the language, and also from its complement $\bar{L}:=\left(\Sigma^{*} \backslash\{\varepsilon\}\right) \backslash L$. As acceptors for such languages, we consider games over an observation alphabet $\Gamma \supseteq \Sigma$, and we assume that no observation sequence in $\Sigma^{+}$is 


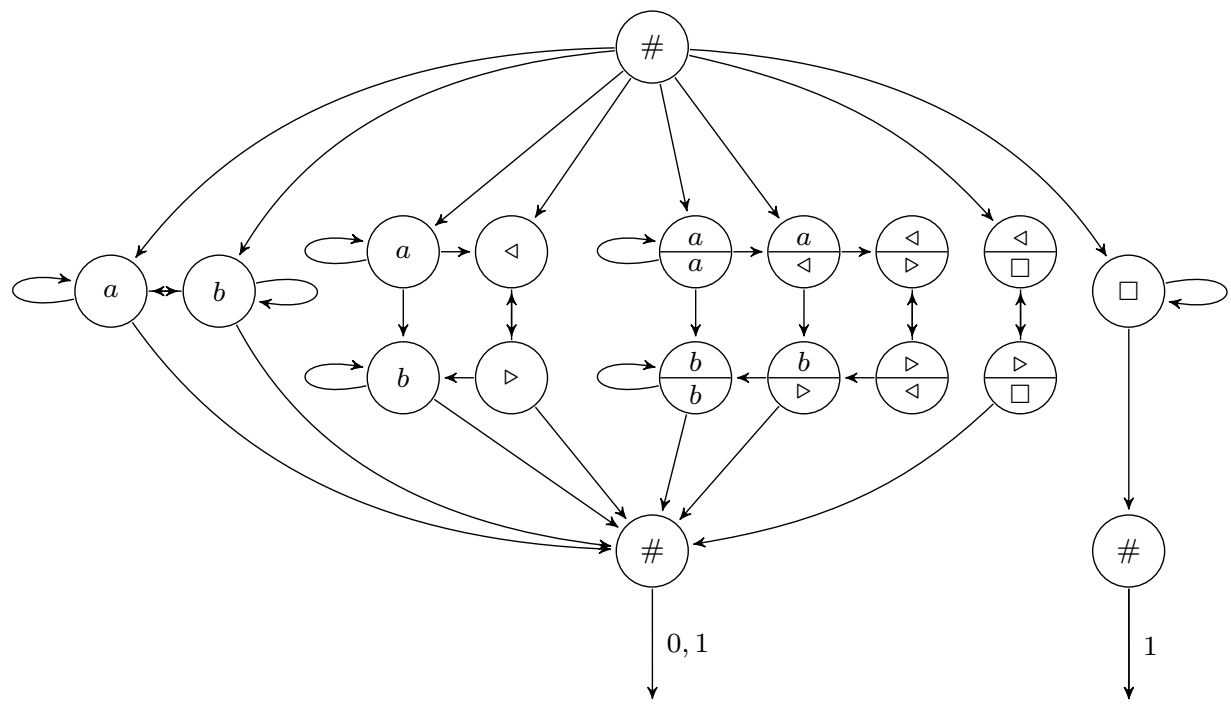

Figure 1. A consensus game acceptor

omitted: for every word $w \in \Sigma^{+}$, and each player $i$, there exists a play $\pi$ that yields the observation sequence $\beta^{i}(\pi)=w$.

Given a consensus game acceptor $G$, we associate to every observationbased strategy $s \in S^{1}$ of the first player, the language $L(s):=\{w \in$ $\left.\Sigma^{*} \mid s(w)=1\right\}$. We say that the game $G$ covers a language $L \subseteq \Sigma^{*}$, if $G$ is solvable and

- $L=L(s)$, for some winning strategy $s \in S^{1}$, and

- $L \subseteq L(s)$, for every winning strategy $s \in S^{1}$.

If, moreover, $L=L(s)$ for every winning strategy in $G$, we say that $G$ characterises $L$. In this case, all winning strategies map $L$ to 1 and $\bar{L}$ to 0 .

Notice that every solvable game covers a unique language $L$ over the full observation alphabet $\Gamma$. With respect to a given terminal alphabet $\Sigma \subseteq \Gamma$, the covered language is hence $L \cap \Sigma^{*}$. For instance, the consensus game acceptor represented in Figure 1 covers the language $\left\{a^{n} b^{n} \mid n \in \mathbb{N}\right\}$ over $\{a, b\}$. To characterise a language rather than covering it, we need to add constraints that require to reject inputs.

Given two games $G, G^{\prime}$, we define the union $G \cup G^{\prime}$ as the consensus game obtained by taking the disjoint union of $G$ and $G^{\prime}$ and identifying the initial states. Then, winning strategies of the component games can be turned into winning strategies of the composite game, if they agree on the observation sequences over the common alphabet.

Lemma 1. Let $G, G^{\prime}$ be two consensus games over observation alphabets $\Gamma$, $\Gamma^{\prime}$. Then, an observation-based strategy $r$ is winning in $G \cup G^{\prime}$ if, and only 
if, there exist observation-based winning strategies $s, s^{\prime}$ in $G, G^{\prime}$ that agree with $r$ on $\Gamma^{*}$ and on $\Gamma^{*}$, respectively.

Proof. Clearly, if $r$ is a winning strategy in $G \cup G^{\prime}$, then its restrictions $s, s^{\prime}$ to $\Gamma^{*}$ and $\Gamma^{*}$ are winning strategies in $G$ and $G^{\prime}$. For the converse, let $s, s^{\prime}$ be two observation-based winning strategies in $G, G^{\prime}$ that agree on $\left(\Gamma \cap \Gamma^{\prime}\right)^{*}$. Notice that every observation sequence in the conjunction $G \cup G^{\prime}$ is either included in $\Gamma^{*}$ or in $\Gamma^{* *}$, as a play that enters $G$ at the initial state can never reach $G^{\prime}$ and vice versa. Therefore, the function $r:\left(\Gamma \cup \Gamma^{\prime}\right)^{*} \rightarrow\{0,1\}$ that agrees with $s$ on $\Gamma^{*}$ and with $s^{\prime}$ on $\Gamma^{*}$ determines a winning strategy in the acceptor game $G \cup G^{\prime}$.

Whenever a language and its complement are covered by two consensus games, we can construct a new game that characterises the language. The construction involves inverting the decisions in a game, that is, replacing the admissible decisions for every final state $v \in V$ with $\Omega(v)=\{0\}$ by $\Omega(v):=$ $\{1\}$ and vice versa; final states $v$ with $\Omega(v)=\{0,1\}$ remain unchanged.

Lemma 2. Suppose two consensus games $G, G^{\prime}$ cover a language $L \subseteq \Sigma^{*}$ and its complement $\bar{L}$, respectively. Let $G^{\prime \prime}$ be the game obtained from $G^{\prime}$ by inverting the admissible decisions. Then, the game $G \cup G^{\prime \prime}$ characterises $L$.

Proof. Let $G, G^{\prime}$ be two acceptor games that cover $L$ and $\bar{L}$. Witout loss of generality, we assume that the observation alphabets $\Gamma, \Gamma^{\prime}$ intersect only on $\Gamma \cap \Gamma^{\prime}=\Sigma$; other common observations may be renamed. We need to show that the union $G \cup G^{\prime \prime}$ is solvable and that every winning strategy $r$ of Player 1 satisfies $L=L(r)$.

For the first point, let $s, s^{\prime}$ be two observation-based winning strategies for Player 1 in $G, G^{\prime}$ such that $L(s)=L$ and $L\left(s^{\prime}\right)=\bar{L}$. Then, the strategy $s^{\prime \prime}$ for $G^{\prime \prime}$ with $s^{\prime \prime}(\pi):=1-s^{\prime}(\pi)$ for any play $\pi$ is winning, since $s^{\prime}$ is winning in $G^{\prime}$ and the admissible decisions are inverted. Moreover, $s$ and $s^{\prime \prime}$ agree on all sequences of observations in $\Sigma^{*}$, so they admit a common extension $r$ to $G \cup G^{\prime \prime}$ that determines a winning strategy, according to Lemma 1 .

For the second point, consider an arbitrary winning strategy $r$ in the composite game $G \cup G^{\prime \prime}$. Then, the restrictions of $r$ to $\Gamma^{*}$ and $\Gamma^{*}$ are winning in $G$ and $G^{\prime \prime}$, respectively. This implies, for all $w \in \Sigma^{*}$, that $r(w)=1$ if $w \in L$, and $r(w)=0$ otherwise: the former because $G$ covers $L$, and the latter because $G^{\prime}$ covers $\bar{L}$ and the decisions in $G^{\prime \prime}$ are inverted. Hence, we have $L(r)=L$ for every winning strategy $r$ in the game $G \cup G^{\prime \prime}$, which thus characterises $L$.

3.1. Domino frontier languages. We use domino systems as an alternative to encoding machine models and formal grammars (See [25] for a survey.). A domino system $\mathcal{D}=\left(D, E_{h}, E_{v}\right)$ is described by a finite set of dominoes together with a horizontal and a vertical compatibility relation $E_{h}, E_{v} \subseteq D \times D$. The generic domino tiling problem is to determine, for a given system $\mathcal{D}$, whether copies of the dominoes can be arranged to tile a 
given space in the discrete grid $\mathbb{Z} \times \mathbb{Z}$, such that any two vertically or horizontally adjacent dominoes are compatible. Here, we consider finite rectangular grids $Z(\ell, m):=\{0, \ldots, \ell+1\} \times\{0, \ldots, m\}$, where the first and last column, and the bottom row are distinguished as border areas. Then, the question is whether there exists a tiling $\tau: Z(\ell, m) \rightarrow D$ that assigns to every point $(x, y) \in Z(\ell, m)$ a domino $\tau(x, y) \in D$ such that:

- if $\tau(x, y)=d$ and $\tau(x+1, y)=d^{\prime}$ then $\left(d, d^{\prime}\right) \in E_{h}$, and

- if $\tau(x, y)=d$ and $\tau(x, y+1)=d^{\prime}$ then $\left(d, d^{\prime}\right) \in E_{v}$.

The Border-Constrained Corridor tiling problem takes as input a domino system $\mathcal{D}$ with two distinguished border dominoes \# and $\square$, together with a sequence $w=w_{1} w_{2} \ldots w_{\ell}$ of dominoes $w_{i} \in D$, and asks whether there exists a height $m$ such that the rectangle $Z(\ell, m)$ allows a tiling $\tau$ with $w$ in the top row, \# in the first and last column, and $\square$ in the bottom row:

- $\tau(i, 0)=w_{i}$, for all $i=1, \ldots, \ell$;

- $\tau(0, y)=\tau(\ell+1, y)=\#$, for all $y=0, \ldots, m-1$;

- $\tau(x, m)=\square$, for all $x=1, \ldots, \ell$.

Domino systems can be used to recognise formal languages. For a domino system $\mathcal{D}$ with side and bottom border dominoes as above, the frontier language $L(\mathcal{D})$ is the set of words $w \in D^{*}$ that yield positive instances of the border-constrained corridor tiling problem. We use the following correspondence between context-sensitive languages and domino systems established by Latteux and Simplot.

Theorem 3 (14, 15). For every context-sensitive language $L \subseteq \Sigma^{*}$, we can effectively construct a domino system $\mathcal{D}$ over a set of dominoes $D \supseteq \Sigma$ with frontier language $L(\mathcal{D})=L$.

Figure 2 describes a domino system for recognising the language $a^{n} b^{n}$ also covered by the game in Figure 1. In the following, we show that domino systems can generally be described in terms of consensus game acceptors.

3.2. Uniform encoding of domino problems in games. Game formulations of domino tiling problems are standard in complexity theory, going back to the early work of Chlebus [7]. However, these reductions are typically non-uniform: they construct for every input instance consisting of a domino system together with a border constraint a different game which depends, in particular, on the size of the constraint. Here, we use imperfect information to define a uniform reduction that associates to a fixed domino system $\mathcal{D}$ a game $G(\mathcal{D})$, such that for every border constraint $w$, the question whether $\mathcal{D}, w$ allows a correct tiling is reduced to the question of whether decision 1 is safe in a certain play associated to $w$ in $G(\mathcal{D})$.

Proposition 3.1. For every domino system D, we can construct, in polynomial time, a consensus game acceptor that covers the frontier language of $D$. 


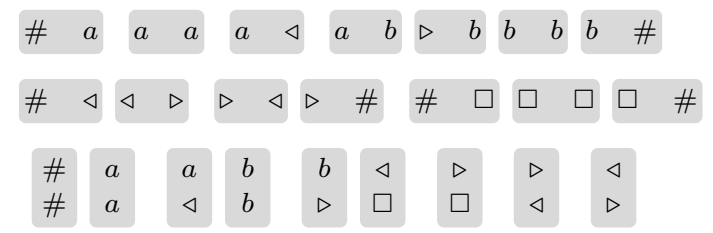

(A) domino system for $a^{n} b^{n}$

$\begin{array}{llllllll}\# & a & a & a & b & b & b & \# \\ \# & a & a & \triangleleft & \triangleright & b & b & \# \\ \# & a & \triangleleft & \triangleright & \triangleleft & \triangleright & b & \# \\ \# & \triangleleft & \triangleright & \triangleleft & \triangleright & \triangleleft & \triangleright & \# \\ \# & \square & \square & \square & \square & \square & \square & \#\end{array}$

(B) tiling $a^{3} b^{3}$

Figure 2. Characterising a language with dominoes

Proof. Let us fix a domino system $\mathcal{D}=\left(D, E_{h}, E_{v}\right)$ with a left border domino \# and a bottom domino $\square$. We construct a consensus game $G$ for the alphabet $\Sigma:=D \backslash\{\#, \square\}$ to cover the frontier language $L(\mathcal{D})$. There are domino states of two types: singleton states $d$ for each $d \in D \backslash\{\#\}$ and pair states $(d, b)$ for each $(d, b) \in E_{v}$. At singleton states $d$, the two players receive the same observation $d$. At states $(d, b)$, the first player observes $d$ and the second player $b$. The domino states are connected by transitions $d \rightarrow d^{\prime}$ for every $\left(d, d^{\prime}\right) \in E_{h}$, and $(d, b) \rightarrow\left(d^{\prime}, b^{\prime}\right)$ whenever $\left(d, d^{\prime}\right)$ and $\left(b, b^{\prime}\right)$ are in $E_{h}$. There is an initial state $v_{0}$ and two final states $\widehat{z}$ and $z$, all associated to the the observation \# for the border domino. From $v_{0}$ there are transitions to all compatible domino states $d$ with $(\#, d) \in E_{h}$, and all pair states $(d, b)$ with $(\#, d)$ and $(\#, b) \in E_{h}$. Conversely, the final state $z$ is reachable from all domino states $d$ with $(d, \#) \in E_{h}$, and all pair states $(d, b)$ with $(d, \#)$ and $(b, \#) \in E_{h}$; the final $\widehat{z}$ is reachable only from the singleton bottom domino state $\square$. Finally, admissible decisions are $\Omega(z)=\{0,1\}$ and $\Omega(\hat{z})=\{1\}$. Clearly, $G$ is a consensus game, and the construction can be done in polynomial time.

Note that any sequence $x=d_{1} d_{2} \ldots d_{\ell} \in D^{\ell}$ that forms a horizontally consistent row in a tiling by $\mathcal{D}$ corresponds in the game to a play $\pi_{x}=$ $v_{0} d_{1} d_{2} \ldots d_{\ell}, z$ or $\pi_{x}=v_{0} \square^{\ell} \widehat{z}$. Conversely, every play in $G$ corresponds either to one possible row, in case Input chooses a single domino in the first transition, or to two rows, in case it chooses a pair. Moreover, a row $x$ can appear on top of a row $y=b_{1} b_{2} \ldots b_{\ell} \in D^{\ell}$ in a tiling if, and only if, there exists a play $\rho$ in $G$ such that $\pi_{x} \sim^{1} \rho \sim^{2} \pi_{y}$, namely $\rho=$ $v_{0}\left(d_{1}, b_{1}\right)\left(d_{2}, b_{2}\right) \ldots\left(d_{\ell}, b_{\ell}\right) z$.

Now, we claim that at an observation sequence $\pi=w$ for $w \in \Sigma^{\ell}$ the decision 0 is safe if, and only if, there exists no correct corridor tiling by $\mathcal{D}$ with $w$ in the top row. According to our remark, there exists a correct tiling of the corridor with top row $w$, if and only if, there exists a sequence of rows corresponding to plays $\pi_{1}, \ldots, \pi_{m}$, and a sequence of witnessing plays $\rho_{1}, \ldots, \rho_{m-1}$ such that $w=\pi_{1} \sim^{1} \rho_{1} \sim^{2} \pi_{2} \ldots \sim^{1} \rho_{m-1} \sim^{2} \pi_{m}=\square^{\ell}$. However, the decision 0 is unsafe in the play $\square^{\ell}$ and therefore at $w$ as well. Hence, every winning strategy $s$ for $G$ must prescribe $s(w)=1$, for every word $w$ in the frontier language of $\mathcal{D}$, meaning that $L(s) \subseteq L(\mathcal{D})$. 
Finally, consider the mapping $s: D^{*} \rightarrow A$ that prescribes $s(w)=1$ if, and only, if $w \in L(\mathcal{D})$. The observation-based strategy $s$ in the consensus game $G$ is winning since $s\left(\square^{*}\right)=1$, and it witnesses the condition $L(s)=L(\mathcal{D})$. This concludes the proof that the constructed consensus game $G$ covers the frontier language of $\mathcal{D}$.

\section{Characterising COnteXt-Sensitive languages}

Our first result establishes a correspondence between context-sensitive languages and consensus games.

Theorem 4. For every context-sensitive language $L \subseteq \Sigma^{*}$, we can construct effectively a consensus game acceptor that characterises $L$.

Proof. Let $L \subseteq \Sigma^{*}$ be an arbitrary context-sensitive language, represented, e.g., by a linear-bounded automaton. By Theorem 3, we can effectively construct a domino system $\mathcal{D}$ with frontier language $L$. Further, by Proposition 3.1, we can construct a consensus game $G$ that covers $L(\mathcal{D})=L$. Due to the Immerman-Szelepcsényi Theorem, context-sensitive languages are effectively closed under complement, so we can construct a consensus game $G^{\prime}$ that covers $\Sigma^{*} \backslash L$ following the same procedure. Finally, we combine the games $G$ and $G^{\prime}$ as described in Lemma 2 to obtain a consensus game that characterises $L$.

One interpretation of the characterisation is that, for every context-sensitive language, there exists a consensus game that is as hard to play as it is to decide membership in the language. On the one hand, this implies that winning strategies for consensus games are in general PSPACE-hard. Indeed, there are instances of consensus games that admit winning strategies, however, any machine that computes the decision to take in a play requires space polynomial in the length of the play.

Theorem 5. There exists a solvable consensus game for which every winning strategy is PSPACE-hard.

Proof. There exist context-sensitive languages with a PSPACE-hard word problem [13]. Let us fix such a language $L \subseteq \Sigma^{*}$ together with a consensus game $G$ that characterises it, according to Theorem 4. This is a solvable game, and every winning strategy can be represented as an observationbased strategy $s$ for the first player. Then, the membership problem for $L$ reduces (in linear time) to the problem of deciding the value of $s$ in a play in $G$ : For every input word $w \in \Sigma^{*}$, we have $w \in L$ if, and only if, $s(w)=1$. In conclusion, for every winning strategy $s$ in $G$, it is PSPACE-hard to decide whether $s(\pi)=1$.

On the other hand, it follows that determining whether a consensus game admits a winning strategy is no easier than solving the emptiness problem of context-sensitive languages, which is well known to be undecidable. 
Theorem 6. The question whether a consensus game admits a winning strategy is undecidable.

Proof. We reduce the emptiness problem for a context-sensitive grammar to the solvability problem for a consensus game. For an arbitrary contextsensitive language $L \in \Sigma^{*}$ given as a linear bounded automaton, we construct a consensus game $G$ that characterises $L$, in polynomial time, according to Theorem 4. Additionally, we construct a consensus game $G^{\prime}$ that characterises the empty language over $\Sigma^{*}$ : this can be done, for instance, by connecting a clique over letters in $\Sigma$ observable for both players to a final state at which only the decision 0 is admissible. Now, for any word $w \in \Sigma^{*}$, the game $G^{\prime}$ requires decision 0 at every observation sequences $w \in \Sigma^{*}$, whereas $G$ requires decision 1 whenever $w \in L$. Accordingly, the consensus game $G \cup G^{\prime}$ is solvable if, and only if, $L$ is empty. As the emptiness problem for context-sensitive languages is undecidable [13], it follows that the solvability problem is undecidable for consensus game acceptors.

We have seen that every context-sensitive language corresponds to a consensus game acceptor such that language membership tests reduce to winning strategy decisions in a play. Conversely, every solvable game admits a winning strategy that is the characteristic function of some context-sensitive language. Intuitively, a strategy should prescribe 0 at a play $\pi$ whenever there exists a connected play $\pi^{\prime}$ at which 0 is the only admissible decision. Whether this is the case can be verified by a nondeterministic machine using space linear in the length of the play $\pi$.

Theorem 7. Every solvable consensus game admits a winning strategy that is implementable by a nondeterministic linear bounded automaton.

\section{Consensus and iterated transductions}

Our aim in the following is to investigate how the structure of a consensus game relates to the complexity of the described language which, in turn, determines the complexity of winning strategies. Towards this, we view games as finite-state automata representing the relation between the observation sequences received by the players and the admissible decisions.

A synchronous transducer is a two-tape automaton $\left(Q, \Gamma, \Delta, q_{0}, F\right)$ over an alphabet $\Gamma$, with state set $Q$, a transition relation $\Delta \subseteq Q \times \Gamma \times \Gamma \times Q$ labelled by pairs of letters, an initial state $q_{0} \in Q$ and a non-empty set $F \subseteq Q$ of final states; in contrast to games, final states of transducers may have outgoing transitions. We write $p \stackrel{a \mid b}{\longrightarrow} q$ to denote a transition $(p, a, b, q) \in \Delta$. An accepting run of the transducer is a path $\rho=q_{0} \stackrel{a_{1} \mid b_{1}}{\longrightarrow}$ $q_{1} \stackrel{a_{2} \mid b_{2}}{\longrightarrow} \ldots \stackrel{a_{n} \mid b_{n}}{\longrightarrow} q_{n}$ that follows transitions in $\Delta$ starting from the initial state $q_{0}$ and ending at a final state $q_{n} \in F$. The label of the run is the pair of words $\left(a_{1} \ldots a_{n}, b_{1}, \ldots b_{n}\right)$. A pair of words $\left(w, w^{\prime}\right) \in \Gamma^{*} \times \Gamma^{*}$ is accepted by the transducer if it is the label of some accepting run. The relation recognised 
by the transducer is the set $R \subseteq \Gamma^{*} \times \Gamma^{*}$ of accepted pairs of words. A relation is synchronous if it is recognised by a synchronous transducer. In general, we do not distinguish notationally between transducers and the relation they recognise. For background on synchronous, or letter-to-letter, transducers, we refer to the survey [3] of Berstel and to Chapter IV in the book 22] of Sakarovitch.

Given a consensus game acceptor $G=\left(V, E, \beta^{1}, \beta^{2}, v_{0}, \Omega\right)$ over an observation alphabet $\Gamma$, we define the seed of $G$ to be the triple $\left(R, L_{\mathrm{acc}}, L_{\mathrm{rej}}\right)$ consisting of the relation $R:=\left\{\left(\beta^{1}(\pi), \beta^{2}(\pi)\right) \in(\Gamma \times \Gamma)^{*} \mid \pi\right.$ play in $\left.G\right\}$ together with the languages $L_{\text {acc }} \subseteq \Gamma^{*}$ and $L_{\text {rej }} \subseteq \Gamma^{*}$ of observation sequences $\beta^{1}(\pi)$ on plays $\pi$ in $G$ with $\Omega(\pi)=\{1\}$ and $\Omega(\pi)=\{0\}$, respectively. The seed of any finite game can be represented by finite-state automata.

Lemma 8. For every consensus game, the seed languages $L_{\mathrm{acc}}, L_{\mathrm{rej}}$ are regular and the seed relation $R$ is recognised by a synchronous transducer.

Proof. We construct automata from the game graph by moving observations from each state to the incoming transitions. Let $G=\left(V, E, \beta, v_{0}, \Omega\right)$ be a consensus game over an observation alphabet $\Gamma$, and let $\left(R, L_{\mathrm{acc}}, L_{\mathrm{rej}}\right)$ be its seed. We define three automata over the alphabet $\Gamma$, on the subset of $V$ consisting of non-final game states and with initial state $q_{0}=v_{0}$.

The automata for the seed languages $L_{\text {acc }}$ and $L_{\text {rej }}$ allow transitions $u \stackrel{a}{\rightarrow} v$ if $(u, v) \in E$ and $\beta^{1}(v)=a$. The set of final states consists of all game states $v$ with an outgoing transition $\left(v, v^{\prime}\right) \in E$ to some final state with $\Omega\left(v^{\prime}\right)=\{1\}$ for $L_{\mathrm{acc}}$, and with $\Omega\left(v^{\prime}\right)=\{0\}$ for $L_{\mathrm{rej}}$. Then, for all words $a_{1} \ldots a_{n} \in \Gamma^{*}$, accepting runs $v_{0} \stackrel{a_{1}}{\longrightarrow} v_{1} \stackrel{a_{2}}{\longrightarrow} \ldots \stackrel{a_{n}}{\longrightarrow} v_{n}$ of the automata $L_{\text {acc }}$ and $L_{\text {rej }}$ correspond to plays $\pi:=v_{0} v_{1} \ldots v_{n} v^{\prime}$ with observations $\beta^{1}(\pi)=$ $a_{1} \ldots a_{n}$ such that $\Omega(\pi)=\{1\}$ and $\Omega(\pi)=0$, respectively. Hence, the automata recognise $L_{\mathrm{acc}}$ and $L_{\mathrm{rej}}$.

Similarly, the transducer for the seed relation has transitions $u \stackrel{a \mid b}{\longrightarrow} v$ whenever $(u, v) \in E$ with $\beta^{1}(v)=a, \beta^{2}(v)=b$, and its final states are states $v \in V$ with an outgoing transition $\left(v, v^{\prime}\right) \in E$ to some terminal state $v^{\prime}$ in $G$. Then, for any pair of words $\left(a_{1} \ldots a_{n}, b_{1} \ldots b_{n}\right) \in \Gamma^{*} \times \Gamma^{*}$, there exists an accepting transducer run $v_{0} \stackrel{a_{1} \mid b_{1}}{\longrightarrow} v_{1} \stackrel{a_{2} \mid b_{2}}{\longrightarrow} \ldots \stackrel{a_{n} \mid b_{n}}{\longrightarrow} v_{n}$ if, and only if, there exists a play $\pi=v_{0}, v_{1}, \ldots, v_{n}, v^{\prime}$ with $\beta^{1}(\pi)=a_{1} \ldots a_{n}, \beta^{2}\left(\pi^{\prime}\right)=b_{1} \ldots b_{n}$, for some final game state $v^{\prime}$. So, the transducer recognises the seed relation $R$, as intended.

Conversely, we can turn synchronous transducers and automata over matching alphabets into games.

Lemma 9. Given a synchronous relation $R \subseteq \Gamma^{*} \times \Gamma^{*}$ and two disjoint regular languages $L_{\mathrm{acc}}, L_{\mathrm{rej}} \subseteq \Gamma^{*}$, we can construct a consensus game with seed $\left(R, L_{\text {acc }}, L_{\text {rej }}\right)$.

Proof. Let us consider a synchronous transducer $\mathcal{R}$ and two word automata $\mathcal{A}, \mathcal{B}$ that recognise $R, L_{\text {acc }}$ and $L_{\text {rej }}$, respectively. We assume that the word 
automata are deterministic, with transition functions $\delta^{\mathcal{A}}: Q^{\mathcal{A}} \times \Gamma \rightarrow Q^{\mathcal{A}}$ and $\delta^{\mathcal{B}}: Q^{\mathcal{B}} \times \Gamma \rightarrow Q^{\mathcal{B}}$. To avoid confusion, we label the components of each automaton with its name and write $p \frac{a}{\mathcal{R}} q$ for the transitions in $\mathcal{R}$.

We construct a game $G$ over the observation alphabet $\Gamma \cup\{\#\}$ with states formed of three components: a transducer transition $p \underset{\mathcal{R}}{\stackrel{a \mid b}{\longrightarrow}} q$, a state of $\mathcal{A}$, and one of $\mathcal{B}$. The game transitions follow the adjacency graph of the transducer in the first component and update the state of $\mathcal{A}$ and $\mathcal{B}$ in the second and third component according to the observation $a$ of player 1 in the first component. More precisely, the set of game states is $V:=\Delta \times$ $Q^{\mathcal{A}} \times Q^{\mathcal{B}} \cup\left\{v_{0}, v_{\text {acc }}, v_{\text {rej }}, v_{=}\right\}$, where $v_{0}$ is a fresh initial state whereas $v_{\text {acc }}$, $v_{\text {rej }}$ and $v_{=}$are final states. Game transitions lead from the initial state $v_{0}$ to all states $\left(q_{0}^{\mathcal{R}} \underset{\mathcal{R}}{\stackrel{a \mid b}{\longrightarrow}} q, q_{0}^{\mathcal{A}}, q_{0}^{\mathcal{B}}\right)$; for each pair of incident transducer transitions $e:=p \underset{\mathcal{R}}{\stackrel{a \mid b}{\rightarrow}} q$ and $e^{\prime}:=q \underset{\mathcal{R}}{\stackrel{a^{\prime} \mid b^{\prime}}{\longrightarrow}} q^{\prime}$, and for all automata states $q^{\mathcal{A}} \in Q^{\mathcal{A}}, q^{\mathcal{B}} \in$ $Q^{\mathcal{B}}$, there is a game transition from $\left(e, q^{\mathcal{A}}, q^{\mathcal{B}}\right)$ to $\left(e^{\prime}, \delta^{\mathcal{A}}\left(q^{\mathcal{A}}, a\right), \delta^{\mathcal{A}}\left(q^{\mathcal{B}}, a\right)\right)$; finally, from any state $\left(p \underset{\mathcal{R}}{\stackrel{a \mid b}{\longrightarrow}} q, q^{\mathcal{A}}, q^{\mathcal{B}}\right)$ with $q \in F^{\mathcal{R}}$ there is a transition to $v_{\text {acc }}$ if $q^{\mathcal{A}} \in F^{\mathcal{A}}$, to $v_{\text {rej }}$ if $q^{\mathcal{B}} \in F^{\mathcal{B}}$, and otherwise to $v_{=}$. The observation at state $\left(p \underset{\mathcal{R}}{\stackrel{a \mid b}{\longrightarrow}} q, q^{\mathcal{A}}, q^{\mathcal{B}}\right)$ is $(a, b)$; at the initial and the final states both players observe \#. Admissible decisions are $\Omega\left(v_{\text {acc }}\right)=\{1\}, \Omega\left(v_{\text {rej }}\right)=\{0\}$, and $\Omega\left(v_{=}\right)=\{0,1\}$.

Now, for any play $\pi$, the first component corresponds to an accepting run of $\mathcal{R}$ on the pair of observation sequences $\left(\beta^{1}(\pi), \beta^{2}(\pi)\right)$, whereas the second and third components correspond to runs of $\mathcal{A}$ and $\mathcal{B}$ on $\beta^{1}(\pi)$ which are accepting if $\Omega(\pi)=\{1\}$ and $\Omega(\pi)=\{0\}$, respectively. Accordingly, the game $G$ has seed $\left(R, L_{\mathrm{acc}}, L_{\mathrm{rej}}\right)$.

Thanks to the translation between games and automata, we can reason about games in terms of elementary operations on their seed. Our notation is close to the one of Terlutte and Simplot 24. Given a relation $R \subseteq$ $\Gamma^{*} \times \Gamma^{*}$, the inverse relation is $R^{-1}:=\left\{(x, y) \in \Gamma^{*} \times \Gamma^{*} \mid(y, x) \in R\right\}$. The composition of $R$ with a relation $R^{\prime} \in \Gamma^{*}$ is $R R^{\prime}:=\{(x, y) \in(\Gamma \times$ $\Gamma)^{*} \mid(x, z) \in R$ and $(z, y) \in R^{\prime}$ for some $\left.z \in \Gamma^{*}\right\}$. For a language $L \subseteq \Gamma^{*}$, we write $R L:=\left\{x \in \Gamma^{*} \mid(x, y) \in R\right.$ and $\left.y \in L\right\}$. For a subalphabet $\Sigma \subseteq \Gamma$, we denote the identity relation by $\left(\cap \Sigma^{*}\right):=\left\{(x, x) \in \Sigma^{*} \times \Sigma^{*}\right\}$. The power $R^{k}$ of $R$ is defined by $R^{0}:=(\cap \Gamma)$, and $R^{k+1}:=R^{k} R$ for $k>0$. Finally, the iteration of $R$ is $R^{*}:=\cup_{0<k<\omega} R^{k}$.

One significant relation obtained from the seed transducer $R$ of a game $G$ is the reflection relation $\tau(R):=R R^{-1}$. That is, a word $w \in \Gamma^{*}$ over the observation alphabet is a reflection of $u \in \Gamma^{*}$ if, whenever player 1 observes $u$, player 2 considers it possible that 1 actually observes $w$. Obviously, this relation is reflexive and symmetric. Its transitive closure relates observations received on connected plays. 
Lemma 10. Let $G$ be a consensus game with seed relation $R \subseteq \Gamma^{*} \times \Gamma^{*}$, and let $\tau:=R R^{-1}$ be its reflection. Then,

(i) $\tau=\left\{\left(\beta^{1}(\pi), \beta^{1}\left(\pi^{\prime}\right)\right) \mid\right.$ plays $\pi \sim^{2} \pi^{\prime}$ in $\left.G\right\}$, and

(ii) $\tau^{*}=\left(\cap \Gamma^{*}\right) \cup\left\{\left(\beta^{1}(\pi), \beta^{1}\left(\pi^{\prime}\right)\right) \mid\right.$ plays $\pi \sim^{*} \pi^{\prime}$ in $\left.G\right\}$.

Proof. ( $i$ ) By our definition of the seed relation, for every pair of words $\left(w, w^{\prime}\right) \in R R^{-1}$, there exist plays $\pi, \pi^{\prime}$ such that $\beta^{1}(\pi)=w, \beta^{1}\left(\pi^{\prime}\right)=w^{\prime}$, and $\beta^{2}(\pi)=\beta^{2}\left(\pi^{\prime}\right)$, that is, $\pi \sim^{2} \pi^{\prime}$. Conversely, for any pair of plays $\pi \sim^{2} \pi^{\prime}$, the observation sequences are related by $\left(\beta^{1}(\pi), \beta^{2}(\pi)\right) \in R$ and $\left(\beta^{2}(\pi), \beta^{1}\left(\pi^{\prime}\right)\right)=\left(\beta^{2}\left(\pi^{\prime}\right), \beta^{1}\left(\pi^{\prime}\right)\right) \in R^{-1}$. Hence $\left(\beta^{1}(\pi), \beta^{1}\left(\pi^{\prime}\right)\right) \in R R^{-1}$.

(ii " $\supseteq$ "): Clearly, $\left(\cap \Gamma^{*}\right) \subseteq \tau^{*}$. Further, by definition of connectedness, if $\pi \sim^{*} \pi^{\prime}$, then there exists a sequence of plays $\left(\pi_{\ell}\right)_{\ell<2 k}$ with $\pi_{0}=\pi$, $\pi_{2 k}=\pi^{\prime}$, and $\pi_{\ell} \sim^{1} \pi_{\ell+1} \sim^{2} \pi_{\ell+2}$ for all even $\ell<2 k$. Therefore, the observation sequences

$$
x:=\beta^{1}\left(\pi_{\ell}\right)=\beta^{1}\left(\pi_{\ell+1}\right), \quad y:=\beta^{1}\left(\pi_{\ell+2}\right), \quad \text { and } \quad z:=\beta^{2}\left(\pi_{\ell+1}\right)=\beta^{2}\left(\pi_{\ell+2}\right)
$$

are related by $(x, z) \in R$ and $(z, y) \in R^{-1}$, which means that $(x, y)=$ $\left(\beta^{1}\left(\pi_{\ell}\right), \beta^{1}\left(\pi_{\ell+2}\right)\right) \in R R^{-1}$, for all even $\ell<2 k$. Accordingly, we obtain $\left(\beta^{1}(\pi), \beta^{1}\left(\pi^{\prime}\right)\right) \in\left(R R^{-1}\right)^{*}$.

(ii "C") To show that every pair of distinct words in $\tau^{*}$ can be observed by player 1 on connected plays, we verify by induction on the power $k \geq 1$ that for every pair $\left(w, w^{\prime}\right) \in\left(R R^{-1}\right)^{k}$, there exists a sequence of plays $\pi_{0} \sim^{1} \pi_{1} \sim^{2} \ldots \sim^{2} \pi_{2 k}$ such that $\beta^{1}\left(\pi_{0}\right)=w$ and $\beta^{1}\left(\pi_{2 k}\right)=w^{\prime}$.

The base case for $k=1$ follows from point $(i)$ of the present lemma: if $\left(w, w^{\prime}\right) \in\left(R R^{-1}\right)$, then there exist $\pi \sim^{2} \pi^{\prime}$ with $\beta^{1}(\pi)=w, \beta^{1}\left(\pi^{\prime}\right)=w^{\prime}$, and we set $\pi_{0}=\pi_{1}=\pi$ and $\pi_{2}=\pi^{\prime}$. For the induction step, assume that the hypothesis holds for a power $k \geq 1$ and consider $\left(w, w^{\prime}\right) \in\left(R R^{-1}\right)^{k+1}$. That is, there exists a word $z \in \Gamma^{*}$ such that $(w, z) \in\left(R R^{-1}\right)^{k}$ and $\left(z, w^{\prime}\right) \in$ $\left(R R^{-1}\right)$. The former implies, by induction hypothesis, that we have a chain $\pi_{0} \sim^{1} \pi_{1} \sim^{2} \ldots \sim^{1} \pi_{2 k-1} \sim^{2} \pi_{2 k}$ with $\beta^{1}\left(\pi_{0}\right)=w$ and $\beta^{1}\left(\pi_{2 k}\right)=z$; from the latter it follows, by definition of $R$, that there exist plays $\pi$ and $\pi^{\prime}$ with $\beta^{1}(\pi)=z=\beta^{1}\left(\pi_{2 k}\right), \beta^{2}(\pi)=\beta^{2}\left(\pi^{\prime}\right)$, and $\beta^{1}\left(\pi^{\prime}\right)=w^{\prime}$. Therefore, we can prolong the witnessing chain by setting $\pi_{2 k+1}:=\pi, \pi_{2 k+2}:=\pi^{\prime}$, which concludes the induction argument.

The consensus condition requires decisions to be invariant under the reflection relation. This yields the following characterisation of winning strategies.

Lemma 11. Let $G$ be a consensus game with seed $\left(R, L_{\mathrm{acc}}, L_{\mathrm{rej}}\right)$ over an alphabet $\Gamma$, and let $\tau:=R R^{-1}$ be its reflection relation. Then, a strategy $s$ : $\Gamma^{*} \rightarrow\{0,1\}$ of player 1 is winning if, and only if, it assigns $s(w)=1$ to every observation sequence $w \in \tau^{*} L_{\text {acc }}$ and $s(w)=0$ to every observation sequence $w \in \tau^{*} L_{\mathrm{rej}}$.

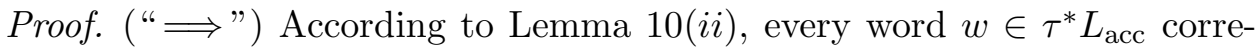
sponds to the observation sequence $\beta^{1}(\pi)=w$ of a play $\pi$ in $G$, and there exists a connected play $\pi^{\prime} \sim^{*} \pi$ with $\pi^{\prime} \in L_{\text {acc }}$. Therefore, any winning 
strategy $s: \Gamma^{*} \rightarrow\{0,1\}$ for player 1 must assign $s(w)=1$ to all $w \in L_{\text {acc }}$ and further, to all $w \in \tau^{*} L_{\text {acc }}$, by the conditions of consensus and indistinguishability. Likewise, it follows that $s(w)=0$ for all $w \in \tau^{*} L_{\text {rej}}$.

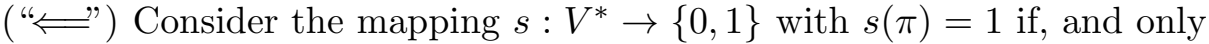
if, $\beta^{1}(\pi) \in \tau^{*} L_{\text {acc. }}$. Then $s$ is a valid strategy, as for all $\pi \sim^{2} \pi^{\prime}$ we have $\left(\beta^{1}(\pi), \beta^{1}\left(\pi^{\prime}\right)\right) \in \tau$ and $\left(\beta^{1}\left(\pi^{\prime}\right), \beta^{1}(\pi)\right) \in \tau$, hence $\beta^{1}(\pi) \in \tau^{*} L_{\text {acc }}$ if, and only if, $\beta^{1}\left(\pi^{\prime}\right) \in \tau^{*} L_{\text {acc }}$. If it is the case that $s(\pi)=0$ for all $\pi \in \tau^{*} L_{\text {rej }}$, that is, $\tau^{*} L_{\mathrm{acc}} \cap \tau^{*} L_{\mathrm{rej}}=\emptyset$, then $s$ is a winning strategy.

As a direct consequence, we can characterise the language defined by a game in terms of iterated transductions.

Theorem 12. Let $G$ be a consensus game with seed $\left(R, L_{\mathrm{acc}}, L_{\mathrm{rej}}\right)$, and let $\Sigma$ be a subset of its alphabet. Then, for the reflection $\tau:=R R^{-1}$, we have:

(i) $G$ is solvable if, and only if, $\tau^{*} L_{\mathrm{acc}} \cap \tau^{*} L_{\mathrm{rej}}=\emptyset$.

(ii) If $G$ is solvable, then it covers the language $\left(\cap \Sigma^{*}\right) \tau^{*} L_{\text {acc }}$.

(iii) If $G$ is solvable and $\left(\cap \Sigma^{*}\right)\left(\tau^{*} L_{\mathrm{acc}} \cup \tau^{*} L_{\mathrm{rej}}\right)=\Sigma^{*}$, then $G$ characterises the language $\left(\cap \Sigma^{*}\right) \tau^{*} L_{\mathrm{acc}}$.

\section{Games for COnteXt-Free languages}

Properties of iterated letter-to-letter transductions, or equivalently, lengthpreserving transductions, have been investigated by Latteux, Simplot, and Terlutte in [16, 24], where it is also shown that iterated synchronous transductions capture context-sensitive languages. Our setting is, however, more restrictive in that games correspond to symmetric transductions. In the following, we investigate a family of consensus game acceptors that captures context-free languages. Since the class is not closed under complement, we will work with the weaker notion of covering a language rather than characterising it. For the language-theoretic discussion, we generally assume that the rejecting seed language $L_{\text {rej }}$ is empty and specify the seed $\left(R, L_{\text {acc }}, \emptyset\right)$ as $\left(R, L_{\text {acc }}\right)$.

Firstly, we remark that regular languages correspond to games where the two players have the same observation function. Clearly, such games admit regular winning strategies whenever they are solvable.

Proposition 6.1. A language $L \subseteq \Sigma^{*}$ is regular if, and only if, it is characterised by a consensus game acceptor where the seed relation is the identity.

Proof. Every regular language $L \subseteq \Sigma^{*}$ is characterised by the game with seed $\left(\left(\cap \Sigma^{*}\right), L, \Sigma^{*} \backslash L\right)$. Conversely, suppose a language $L \subseteq \Sigma^{*}$ is characterised by a game $G$ over an alphabet $\Gamma \supseteq \Sigma$ with seed $\left(\left(\cap \Gamma^{*}\right), L_{\text {acc }}, L_{\text {rej }}\right)$. Then, $G$ also covers $L$ and, by Theorem 12 (ii), it follows that $L=\left(\cap \Sigma^{*}\right)\left(\cap \Gamma^{*}\right)^{*} L_{\text {acc }}=$ $\Sigma^{*} \cap L_{\text {acc }}$. Hence, $L$ is regular.

6.1. Dyck languages. As a next exercise, let us construct games for covering Dyck languages, that is, languages of well-balanced words of brackets; we also allow neutral symbols, which may appear at any position without 
affecting the bracket balance. Our terminal alphabet $A$ consists of an alphabet $B_{n}=\left\{[k,]_{k} \mid 1 \leq k \leq n\right\}$ of $n \geq 1$ matching brackets and a set $C$ of neutral symbols. For a word $w \in A^{*}$ and an index $k$, we denote by $\operatorname{excess}^{k}(w)$ the difference between the number of opening and of closing brackets $[k \text { and }]_{k}$. Then, the Dyck language $D_{A}$ over $A$ consists of the words $w \in A^{*}$ such that, for each kind of brackets $k \in\{1, \ldots, k\}$, $\operatorname{excess}^{k}(w)=0$, whereas for all prefixes $w^{\prime}$ of $w$, $\operatorname{excess}^{k}\left(w^{\prime}\right) \geq 0$.

Given a terminal alphabet $A=B_{n} \cup C$, we define the transducer $R_{n, C}$ over the observation alphabet $\Gamma:=A \cup\{\square\}$ with a set of states $\left\{q_{0}, q_{1}, \ldots, q_{n}\right\}$ among which $q_{0}$ is the initial and the only final state, and with the following two kinds of transitions: copying transitions $q_{0} \stackrel{a \mid a}{\longrightarrow} q_{0}$ for all $a \in \Gamma$, as well as $q_{k} \stackrel{\square}{\longrightarrow} q_{k}$ for every $k \in\{1, \ldots n\}$, and erasing transitions $q_{0} \stackrel{\left[{ }_{k} \mid \square\right.}{\longrightarrow} q_{k}$ and $q_{k} \stackrel{]_{k} \mid \square}{\longrightarrow} q_{0}$ for the brackets of each kind $k$, and $q_{0} \stackrel{c \mid \square}{\longrightarrow} q_{0}$ for each neutral symbol $c \in C$. Essentially, $R_{n, C}$ erases neutral symbols and any innermost pair of brackets.

Lemma 13. The Dyck language over an alphabet $n$ of matching brackets and a set $C$ of neutral symbols is covered by the game with seed $\left(R_{n, C}, \square^{*}\right)$.

Proof. For a terminal alphabet $A$ with partitions $B_{n}, C$ as in the statement, we denote the corresponding Dyck language by $D_{A}$ and the previously defined transduction by $R:=R_{n, C}$. The observation alphabet $\Gamma=B_{n} \cup C \cup\{\square\}$ extends $A$ with an additional neutral symbol $\square$; let $D_{\Gamma} \supseteq D_{A}$ be the Dyck language over this extended alphabet. Consider now the game $G$ over $\Gamma$ with seed $\left(R, \square^{*}\right)$. We using the reflection relation $\tau:=R R^{-1}$ to argue that $D_{A}=\tau^{*} \square^{*}$.

To see that the Dyck language $D_{A}$ is contained in the language $\tau^{*} \square^{*}$ covered by $G$, observe that for every pair of words $u, u^{\prime} \in \Gamma^{*}$ where $u \in D_{\Gamma}$ and $u^{\prime}$ is obtained from $u$ by replacing one innermost pair of matching brackets with $\square$, we have $\left(u, u^{\prime}\right) \in R$. Since the relation $R$ contains the identity on $\Gamma$, it follows that $\left(u, u^{\prime}\right) \subseteq R R^{-1}$, so $\left(u, u^{\prime}\right) \in \tau$. If we set out with an arbitrary word $w \in A^{*}$, first erase all neutral symbols by applying $R$ once, and then repeat applying $R$ to erase an innermost pair of brackets, we end up with $\square^{*}$, hence $w \in \tau^{*} \square^{*}$.

Conversely, to verify that every word in $\tau^{*} \square^{*}$ has well-balanced brackets, we show that $D_{\Gamma}$ is invariant under the transductions $R$ and $R^{-1}$ in the sense that for any pair $(u, w) \in R \cup R^{-1}$, we have $u \in D_{\Gamma}$ if, and only if, $w \in D_{\Gamma}$. Towards this, let us fix an accepting run of $R$ on $u \mid w$ and compare the values $\operatorname{excess}^{k}\left(u^{\prime}\right)$ and $\operatorname{excess}^{k}\left(w^{\prime}\right)$ of its prefixes $u^{\prime} \mid w^{\prime}$, for any $k \geq n$ : the values are equal until a transition $q_{0} \stackrel{{ }_{k} \mid \square}{\longrightarrow} q_{k}$ is taken at some prefix $u^{\prime}\left[{ }_{k} \mid w^{\prime} \square\right.$. Since $q_{k}$ is not final, the run will take the transition $q_{k} \stackrel{]_{k} \mid \square}{\longrightarrow} q_{0}$ at some later position; let $\left.u^{\prime \prime}\right]_{k} \mid w^{\prime \prime} \square$ be the shortest continuation of $u^{\prime} \mid w^{\prime}$ at which $q_{0}$ is reached again. Hence, we set out with $\operatorname{excess}^{k}\left(w^{\prime}\right)=\operatorname{excess}^{k}\left(u^{\prime}\right)$, and also have $\operatorname{excess}^{k}\left(u^{\prime}\right)=\operatorname{excess}^{k}\left(u^{\prime \prime}\right)$, since none of $[k \text { or }]_{k}$ is transduced while looping 
in $q_{k}$; after returning to $q_{0}$, again $\operatorname{excess}^{k}\left(w^{\prime \prime}\right)=\operatorname{excess}^{k}\left(u^{\prime \prime}\right)$, because the opening bracket was matched. So, it is the case that $\operatorname{excess}^{k}\left(u^{\prime}\right) \geq 0$ for all prefixes $u^{\prime}$ of $u$ if, and only if, $\operatorname{excess}^{k}\left(w^{\prime}\right) \geq 0$ for all prefixes $w^{\prime}$ of $w$, which means that $R D_{\Gamma} \subseteq D_{\Gamma}$ and $R^{-1} D_{\Gamma} \subseteq D_{\Gamma}$; the converse inclusions hold because $R$ contains the identity on $\Gamma$. Accordingly, for every sequence $w_{0}, \ldots, w_{\ell}$ of words with $w_{0}=w$ such that $\left(w_{i}, w_{i+1}\right) \in \tau$ for each $i<\ell$, we have $w_{i} \in D_{\Gamma}$ if, and only if, $w_{i+1} \in D_{\Gamma}$. Since $\square^{*} \in D_{\Gamma}$, it follows that $w \in D_{\Gamma}$, for any word $w \in \tau^{*} \square^{*}$. In conclusion, $\left(\cap A^{*}\right) \tau^{*} \square^{*} \subseteq D_{A}$.

6.2. Context-free languages. To extend the game description of Dyck languages to arbitrary context-free languages, we use the Chomsky-Schützenberger representation theorem [8] in the non-erasing variant of proved by Okhotin [18]. A letter-to-letter homomorphism $h: A^{*} \rightarrow \Sigma^{*}$ is a functional synchronous transduction that preserves concatenation, that is, $h(u w)=h(u) h(w)$ for all words $u, w \in A^{*}$. Such a homomorphism is identified by its restriction $f: A \rightarrow \Sigma$ to single letters.

Theorem 14 ([18]). A language $L \subseteq \Sigma^{*}$ is context-free if, and only if, there exists a Dyck language $D_{A}$ over an alphabet $A$ of brackets and neutral symbols, a regular language $M \subseteq A^{*}$, and a letter-to-letter homomorphism $h: A^{*} \rightarrow \Sigma^{*}$, such that $L=h\left(D_{A} \cap M\right)$.

We will show how a game that covers an arbitrary language $L \subseteq A^{*}$ can be extended to cover an homomorphic image of the intersection of $L$ with a regular language. Let $h: A \rightarrow \Sigma$ be a letter-to-letter homomorphism, and let $R$ be a synchronous transducer over an alphabet $\Gamma \supseteq A$. We construct from $R$ a new transducer $R_{h}$ over the enlarged alphabet $\Sigma \cup \Gamma \times A$ by adding a coding cycle. This is done by including a fresh final state $q_{h}$, as well as transitions $q_{0} \stackrel{h(a) \mid(a, a)}{\longrightarrow} q_{h}$ and $q_{h} \stackrel{h(a) \mid(a, a)}{\longrightarrow} q_{h}$ for all $a \in A$, and then relabelling each transition $p \stackrel{a \mid b}{\longrightarrow} q$ of $R$ to $p \stackrel{(a, x) \mid(b, x)}{\longrightarrow} q$, for all $x \in A$. Intuitively, the new transducer duplicates the automaton tapes into two tracks which are both initialised with a homomorphic pre-image $u \in A^{*}$ of a terminal word $w \in \Sigma^{*}$, in a transduction via the coding cycle. The first track is intended to simulate $R$ on the pre-image $u$, whereas the second track stores $u$ : the contents is looped through every other transduction of $R_{h}$ or of its inverse. Notice that every run of $R_{h}$ proceeds either through the new coding cycle, or through the original transducer $R$, in the sense that, for any pair $\left(w, w^{\prime}\right) \in R_{h}$ we have $\left\{w, w^{\prime}\right\} \subseteq \Sigma^{*} \cup(\Gamma \times A)^{*}$.

Lemma 15. Suppose that a game acceptor with seed $\left(R, L_{\mathrm{acc}}\right)$ covers a language $L \subseteq A^{*}$. Let $M \subseteq A^{*}$ be a regular language and let $h: A^{*} \rightarrow$ $\Sigma^{*}$ be a letter-to-letter homomorphism. Then, the game acceptor with seed $\left(R_{h}, L_{\text {acc }} \times M\right)$ covers the language $h(L \cap M)$ over the terminal alphabet $\Sigma$.

Proof. Let $G$ be a game over an alphabet $\Gamma \supseteq A$ with seed $\left(R, L_{\text {acc }}\right)$. Without loss of generality, we assume that $R$ contains the identity on $A$, otherwise we take the reflexive transduction $R R^{-1}$ to obtain the seed of a game that 
covers the same language. Further, let $M \subseteq A^{*}$ be a regular language and let $h: A \rightarrow \Sigma$ represent a letter-to-letter homomorphism as in the statement. We argue for the case where the alphabets $\Sigma$ and $\Gamma$ are disjoint; the general case follows by composition with a relabelling homomorphism. Now, consider the game $G^{\prime}$ with seed transducer $R_{h}$ and accepting language $L_{\text {acc }}^{\prime}:=L_{\text {acc }} \times M$. We denote the reflection relations associated $R$ and $R_{h}$ by $\tau:=R R^{-1}$ and $\tau^{\prime}:=R_{h} R_{h}^{-1}$.

To see that $h(L \cap M)$ is included in the language covered by $G^{\prime}$, consider a word $w=h(u)$ for some $u \in \tau^{*} L_{\text {acc }} \cap M$. By Theorem 12, there exists a witnessing sequence $\left(u_{i}\right)_{i \leq \ell}$ with $u_{0}=u, u_{\ell} \in L_{\text {acc }}$, and $\left(u_{i}, u_{i+1}\right) \in$ $\tau$ for all $i<\ell$. By construction of $R_{h}$, we have $(w,(u, u)) \in R_{h}$ which implies $(w,(u, u)) \in \tau^{\prime}$, thanks to our assumption that $\left(\cap A^{*}\right) \subseteq R$. Since $\left(u_{\ell}, u\right) \in L_{\text {acc }} \times M$, the sequence starting with $w$ and followed by $\left(\left(u_{i}, u\right)\right)_{i<\ell}$ is witnessing that $w \in \tau^{*} L_{\text {acc }}^{\prime}$.

Conversely, consider a word $w \in\left(\cap \Sigma^{*}\right) \tau^{\prime *} L_{\text {acc }}^{\prime}$ and let $\left(w_{i}\right)_{i \leq \ell}$ be a witnessing sequence with $w_{0}=w, w_{\ell} \in L_{\text {acc }}^{\prime}$, and $\left(w_{i}, w_{i+1}\right) \in \tau^{\prime}$ for all $i \leq \ell$. By construction of $R_{h}$, the initial word $w$ is preserved at each term $w_{i}$ of the sequence, in the sense that either $w_{i}=w$, or $w_{i}=\left(u_{i}, x_{i}\right) \in \Gamma^{*} \times A^{*}$ for some $x_{i} \in A^{*}$ such that $w=h\left(x_{i}\right)$. By our assumption that $\Sigma$ and $\Gamma$ are disjoint, we have $w \notin L_{\text {acc }}$, so there exists a last position $k<\ell$ with $w_{k}=w$. As $w \in \Sigma^{*}$ can only be transduced via the coding cycle, it follows that $w_{i+1}=(u, u)$ for some $u \in A^{*}$ with $h(u)=w$. For each following position $i>k$, the terms of the sequence are of the form $w_{i}=\left(u_{i}, u\right)$ for a certain word $u_{i} \in \Gamma^{*}$. Hence the coding cycle cannot be applied and the sequence $\left(u_{i}\right)_{k<i \leq \ell}$ satisfies, $\left(u_{i}, u_{i+1}\right) \in \tau$ for all $i<\ell$. Moreover, $w_{\ell}=\left(u_{\ell}, u\right) \in L_{\text {acc }}^{\prime}=L_{\text {acc }} \times M$. Thus, the sequence witnesses that $u=u_{k+1} \in \tau^{*} L_{\text {acc }} \cap M$, and since $h(u)=w$, it follows that $w \in h(L \cap M)$.

Now, we can construct a game acceptor for covering an arbitrary contextfree language $L \subseteq \Sigma^{*}$ represented as $L=h\left(D_{A} \cap M\right)$ according to Theorem 14, by applying Lemma 15 to the particular case of Dyck languages: we set out with the seed transducer $R_{n, C}$ for the Dyck language $D_{A}$ over the alphabet $A=B_{n} \cup C$ and add a coding cycle for the homomorphism $h$. This yields a transducer $R_{h}$ over the alphabet $\Sigma \cup(A \cup\{\square\}) \times A$ such that the game with seed $\left(R_{h}, \square^{*} \times M\right)$ covers $L$.

The generic construction of $R_{h}$ can be simplified in the case where $R=$ $R_{n, C}$ is the seed transducer of a Dyck language. Notice that, if we start from a word $w \in \Sigma^{*}$, then every distinct word $w^{\prime} \neq w$ reached in the iteration $\left(w, w^{\prime}\right) \in\left(R_{h} R_{h}^{-1}\right)^{*}$ consists only of letters of the form $(x, x)$ or $(\square, x)$ with $x \in B_{n} \cup C$. Hence, the reduced transducer $\hat{R}_{h}$ obtained from $R_{h}$, by restricting to the (subset of transitions labelled with letters in the) subalphabet $\Sigma \cup\{(x, x) \mid x \in A\} \cup\{(\square, x) \mid x \in A\}$ and identifying each pair $(x, x) \in A \times A$ with $x$, is equivalent to $R_{h}$ in the sense that, for every regular language $M \subseteq A^{*}$, the game with seed $\left(\hat{R}_{h}, \square^{*} \times M\right)$ covers the same language over $\Sigma$ as the one with seed $\left(R_{h}, \square^{*} \times M\right)$. In contrast to $R_{h}$, 


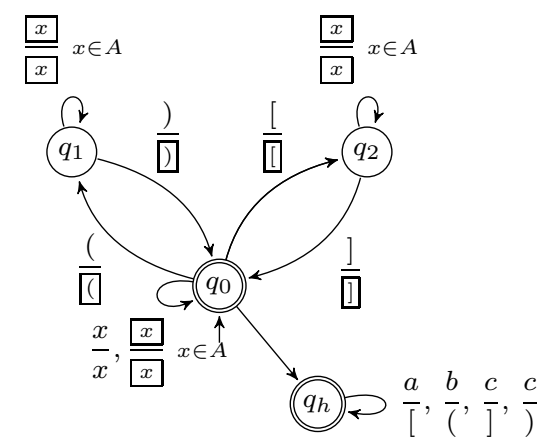

(A) 2-flower

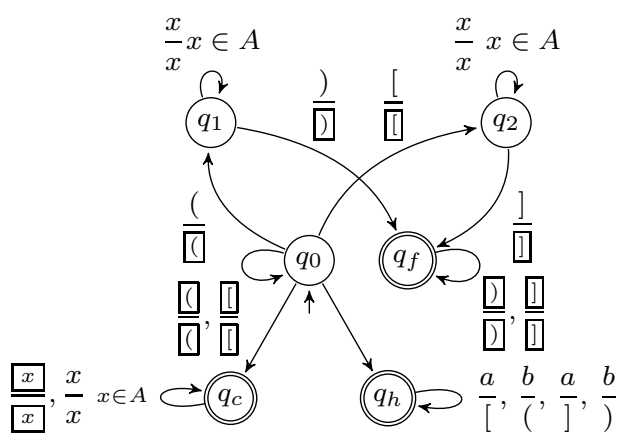

(B) loose flower for palindromes

FiguRE 3. Flower transducers

however, the reduced transducer $\hat{R}_{h}$ has fewer transition and a smaller alphabet, which extends the one of the underlying Dyck language $D_{A}$ only with a neutralised copy of each letter in $A$.

We argue that the shape of the seed constructed above is prototypical for games that cover context-free languages. Therefore, we focus on games with a seed isomorphic to the seed $\left(\hat{R}_{h}, \square^{*} \times M\right)$ obtained for the homomorphic image of a Dyck-language over $n$ bracket pairs intersected with a regular language. An $n$-flower transducer is a transducer $R=\left(Q, \Gamma, \Delta, q_{0}, F\right)$ on a set of states $Q=\left\{q_{0}, q_{1}, \ldots, q_{n}, q_{h}\right\}$ with initial state $q_{0}$ and final state set $F=\left\{q_{0}, q_{h}\right\}$, over an alphabet that can be partitioned into $\Gamma=\Sigma \cup B_{n} \cup$ $C \cup A^{\prime}$ where $B_{n}$ is a set of $n$ matching brackets $\left[{ }_{k},\right]_{k}$ and $A^{\prime}$ is a disjoint copy of $A:=B_{n} \cup C$ associating a neutralised variant $a$ to each letter $a \in A$, such that $\Delta$ contains copying transitions $q_{0} \stackrel{a \mid a}{\longrightarrow} q_{0}$ for all $a \in A \cup A^{\prime}$, and $q_{k} \stackrel{a ! a}{\longrightarrow} q_{k}$ for all $a \in A$ and each $k \in\{1, \ldots n\}$, as well as erasing transitions $q_{0} \stackrel{c \mid \square}{\longrightarrow} q_{0}$ for each $c \in C$, and $q_{0} \stackrel{\left[k_{k} \mid\lfloor\underline{\underline{k}}\right.}{\longrightarrow} q_{k}, q_{k} \stackrel{]_{k} \mid \bigsqcup_{k}}{\longrightarrow} q_{0}$ for each $k$. Furthermore, we require that there is a homomorphism $h: A \rightarrow \Sigma$, such that the remaining transitions of $\Delta$ are coding transitions $q_{0} \stackrel{h(a) \mid a}{\longrightarrow} q_{h}$ and $q_{h} \stackrel{h(a) \mid a}{\longrightarrow} q_{h}$ for all $a \in A$. Finally, a seed $\left(R, L_{\text {acc }}\right)$ is an $n$-flower if $R$ is an $n$-flower transducer and $L_{\text {acc }}$ is a regular language over the alphabet $A^{\prime}$ of its neutralised symbols. An example of a 2-flower transducer is depicted in Figure 3a,

Theorem 16. A language is context-free if, and only if, it is covered by a consensus game acceptor with an $n$-flower seed.

Proof. Let $L \subseteq \Sigma^{*}$ be an arbitrary context-free language. According to the Representation Theorem 14, there exists an alphabet $A$ partitioned into a bracket alphabet $B_{n}$ and a set of neutral symbols $C$, a letter-to-letter homomorphism given by $h: A \rightarrow \Sigma$, and a regular language $M \subseteq A^{*}$, such that $L=h\left(D_{A} \cap M\right)$. With these in hand, we construct the seed transducer 
$R_{n, C}$ for the Dyck language $D_{A}$ as in Lemma 13 and then add a coding cycle $q_{h}$ for the homomorphism $h$. According to Lemma 15, the transducer $R$ constructed in this way together with the seed language $L_{\text {acc }}:=\square^{*} \times M$ describe a game that covers $L$. By reducing $R$ to the alphabet $\Sigma \cup A \cup\{\square\} \times A$, we finally obtain the $n$-flower seed seed $\left(\hat{R}, \square^{*} \times M\right)$ that also covers $L$ over $\Sigma$.

For the converse statement, let $G$ be a consensus game with an $n$-flower seed $\left(R, L_{\text {acc }}\right)$ over an alphabet $\Gamma$. We wish to prove that the language covered by $G$ over $\Gamma$ is context-free. First, we partition $\Gamma$ into an alphabet $\Sigma$, a set $B_{n}$ of $n$ matching brackets, a set $C$ of (prime) neutral symbols, and a neutralised copy $A^{\prime}$ of $A:=B_{n} \cup C$. Let $D_{A}$ be the Dyck language over $A$, and let $h: A \rightarrow \Sigma$ be the letter-to-letter homomorphism determined by the coding cycle in $R$. Then, consider the neutralising letter-to-letter homomorphism $\nu: A \cup A^{\prime} \rightarrow A^{\prime}$ which maps both $a$ and $\square$ to the neutralised copy $\square$, and set $M:=\nu^{-1} L_{\text {acc }}$; as an inverse homomorphic image of a regular language, $M$ is regular. By construction of the flower transducer, we know that, over the alphabet $\Sigma$, the game $G$ covers the context-free language $h\left(D_{A} \cap M\right)$.

To describe the language covered over the full alphabet $\Gamma$, consider the Dyck language $D_{A}^{\prime}$ over the alphabet $A \cup A^{\prime}$ with the same set $B_{n}$ of brackets as $D_{A}$, but with an extended set $C \cup A^{\prime}$ of neutral symbols. We observe that $D_{A}^{\prime}$ is closed under $R$ and $R^{-1}$ in the sense that, for any pair of nonterminal words $w, w^{\prime} \in \Gamma^{*} \backslash \Sigma^{*}$ with $\left(w, w^{\prime}\right) \in R$, we have $w \in D_{A}^{\prime}$ if, and only if, $w^{\prime} \in D_{A}^{\prime}$. Moreover, $\nu(w)=\nu\left(w^{\prime}\right)$ (letters are neutralised, but never forgotten). This implies that, over $\Gamma \backslash \Sigma$, the game $G$ covers the language $D_{A}^{\prime} \cap M$. Since every observation sequence of $G$ is either contained in $\Sigma^{*}$ or in $(\Gamma \backslash \Sigma)^{*}$, it follows that, over the full alphabet $\Gamma$, the consensus game $G$ covers the context-free language $h\left(D_{A} \cap M\right) \cup\left(D_{A}^{\prime} \cap M\right)$.

Notice that without restricting the alphabet of the accepting seed language to neutralised symbols, the flower structure of the transducer alone would not guarantee that the language covered by a game is context-free. For instance, the one-flower transducer over a bracket pair [,] one neutral symbol \#, and their neutralised copies, together with the seed language $L_{\mathrm{acc}}:=\left[^{+} \#[]^{+}\right]^{+}$give rise to a game where the covered language $L$ is not context-free, since the intersection $L \cap[]^{+} \#\left[^{+}\right]^{+}=[]^{n} \#\left[^{n}\right]^{n}$ is not context-free.

Returning to games, the argument from Lemma 11 shows that, given a game with seed $\left(R, L_{\mathrm{acc}}, L_{\mathrm{rej}}\right)$, at every play $\pi$ with observation $\beta^{1}(\pi)$ in the language $L_{1}$ covered by $\left(R, L_{\text {acc }}\right)$ over the full observation alphabet, the only safe decision is 1 , whereas at each play with observations in the language $L_{0}$ covered by $\left(R, L_{\text {rej }}\right)$ the only safe decision is 0 . An observationbased strategy prescribing $s^{1}(\pi):=1$ precisely if $\beta^{1}(\pi) \in L_{1}$ induces a joint strategy that is optimal in the sense that it prescribes a safe decision whenever one exists. Likewise, a strategy that prescribes 0 precisely at sequences in $L_{0}$ is optimal. Optimal strategies are undominated, that is, no 
other strategy wins strictly more plays. Clearly, if a game is solvable, then every optimal strategy is winning.

One consequence of Theorem 16 is that, for games where one of $\left(R, L_{\mathrm{acc}}\right)$ or $\left(R, L_{\text {rej }}\right)$ is an $n$-flower, the set $L_{1}$ or $L_{0}$ is context-free, and therefore recognisable by a nondeterministic push-down automata, which we can construct effectively from the game description. The obtained automaton hence implements an optimal strategy that is indeed a winning strategy, in case the game is solvable. According to Theorem 12, already for games where both $L_{0}$ and $L_{1}$ are context-free, the question of whether a winning strategy exists amounts to solving the disjointness problem for context-free languages and is hence undecidable. Under these circumstances, it is remarkable that we can effectively construct strategies that are optimal and, moreover, winning whenever the game is solvable.

Corollary 17. For any consensus game $G$ with seed $\left(R, L_{\mathrm{acc}}, L_{\mathrm{rej}}\right)$ where either $\left(R, L_{\mathrm{acc}}\right)$ or $\left(R, L_{\mathrm{rej}}\right)$ is an $n$-flower, we can effectively construct a push-down automaton $\mathcal{S}$ that implements an optimal strategy.

\section{Conclusion}

We presented a simple kind of games with imperfect information where constructing optimal strategies requires iterating the (synchronous rational) relation that correlates the observation of players. This establishes a correspondence between winning strategies in games on the one hand, and main classes of formal languages on the other hand. The correspondence leads to several insights on games with imperfect information.

Firstly, we obtain simple examples that illustrate the computational complexity of coordination under imperfect information. The classical constructions for proving that the problem is undecidable in the general case, typically involve an unbounded number of non-trivial decisions by which the players describe configurations of a Turing machine [20, 2, 23]. In contrast, our undecidability argument in Theorem 6 relies on a single simultaneous decision.

Secondly, we identify families of games where optimal strategies exist and can be constructed effectively, but the complexity of the strategic decision necessarily grows with the length of the play. This opens a new perspective for distributed strategy synthesis that departs from the traditional focus on finite-state winning strategies and on game classes on which the existence of such is decidable. In consensus games, the implementation of winning strategies requires arbitrary linear-bounded automata in the general case. However, we also described a structural condition on game graphs that ensures that winning strategies can be implemented by push-down automata.

One challenging objective is to classify games with imperfect information according to the complexity of strategies required for solving them. The insights developed for consensus games allow a few more steps into this 
direction. For instance, games with one-flower seeds cover one-counter languages and therefore admit optimal strategies implemented by one-counter automata. Likewise, we can build up a variant of $n$-flower seeds from Dyck languages restricted to palindromes, as illustrated in Figure 3b, Games with seeds of this kind cover a subclass of linear languages and hence admit optimal strategies implemented by one-turn push-down automata.

\section{REFERENCES}

1. Rajeev Alur, Thomas A. Henzinger, and Orna Kupferman, Alternating-time temporal logic, J. ACM 49 (2002), no. 5, 672-713.

2. Salman Azhar, Gary Peterson, and John Reif, Lower bounds for multiplayer noncooperative games of incomplete information, Journal of Computers and Mathematics with Applications 41 (2001), 957-992.

3. Jean Berstel, Transductions and context-free languages, Teubner, 1979.

4. Dietmar Berwanger and Łukasz Kaiser, Information tracking in games on graphs, Journal of Logic, Language and Information 19 (2010), no. 4, 395-412.

5. Dietmar Berwanger and Marie van den Bogaard, Consensus game acceptors, Proc. of Developments in Language Theory (DLT 2015) (Igor Potapov, ed.), Lecture Notes in Computer Science, vol. 9168, Springer, 2015, pp. 108-119.

6. Ashok K. Chandra, Dexter Kozen, and Larry J. Stockmeyer, Alternation, Journal of the ACM 28 (1981), no. 1, 114-133.

7. Bogdan S. Chlebus, Domino-tiling games, Journal of Computer and System Sciences 32 (1986), no. 3, $374-392$.

8. N. Chomsky and M.P. Schützenberger, The algebraic theory of context-free languages, Computer Programming and Formal Systems, Studies in Logic and the Foundations of Mathematics, vol. 35, Elsevier, 1963, pp. $118-161$.

9. Cynthia Dwork and Yoram Moses, Knowledge and common knowledge in a byzantine environment: Crash failures, Inf. Comput. 88 (1990), no. 2, 156-186.

10. Ronald Fagin, Joseph Y. Halpern, Yoram Moses, and Moshe Y. Vardi, Reasoning about knowledge, MIT Press, 1995.

11. Robert A. Hearn and Erik D. Demaine, Games, puzzles, and computation, A. K. Peters, Ltd., Natick, MA, USA, 2009.

12. David Janin, On the (high) undecidability of distributed synthesis problems, Proc. of Theory and Practice of Computer Science (SOFSEM 2007), Lecture Notes in Computer Science, vol. 4362, Springer, 2007, pp. 320-329.

13. Sige-Yuki Kuroda, Classes of languages and linear-bounded automata, Information and Control 7 (1964), no. 2, 207-223.

14. M. Latteux and D. Simplot, Context-sensitive string languages and recognizable picture languages, Information and Computation 138 (1997), no. 2, 160 - 169.

15. Michel Latteux and David Simplot, Recognizable picture languages and domino tiling, Theoretical Computer Science 178 (1997), no. 12, 275 - 283.

16. Michel Latteux, David Simplot, and Alain Terlutte, Iterated length-preserving rational transductions, Proc. of Mathematical Foundations of Computer Science (MFCS 1998), Springer, 1998, pp. 286-295.

17. Swarup Mohalik and Igor Walukiewicz, Distributed games, Proc. of Foundations of Software Technology and Theoretical Computer Science (FSTTCS 2003), Springer, 2003, pp. 338-351.

18. Alexander Okhotin, Non-erasing variants of the Chomsky-Schützenberger theorem, Proc. of Developments in Language Theory (DLT 2012) (Hsu-Chun Yen and OscarH. Ibarra, eds.), Lecture Notes in Computer Science, vol. 7410, Springer Berlin Heidelberg, 2012, pp. 121-129 (English). 
19. Gary L. Peterson and John H. Reif, Multiple-Person Alternation, Proc 20th Annual Symposium on Foundations of Computer Science, (FOCS 1979), IEEE, 1979, pp. 348363.

20. Amir Pnueli and Roni Rosner, Distributed reactive systems are hard to synthesize, Proc. of Foundations of Computer Science (FoCS 1990), IEEE Computer Society Press, 1990, pp. 746-757.

21. Grzegorz Rozenberg and Arto Salomaa (eds.), Handbook of formal languages, vol. 1: Word, language, grammar, Springer-Verlag New York, Inc., New York, NY, USA, 1997.

22. Jacques Sakarovitch, Elements of automata theory, Cambridge University Press, 2009.

23. Sven Schewe, Distributed synthesis is simply undecidable, Inf. Process. Lett. 114 (2014), no. 4, 203-207.

24. Alain Terlutte and David Simplot, Iteration of rational transductions, Informatique théorique et applications 34 (2000), no. 2, 99-129.

25. Peter van Emde Boas, The convenience of tilings, Complexity, Logic, and Recursion Theory, Lecture Notes in Pure and Applied Mathematics, vol. 18, Marcel Dekker Inc, 1997, pp. 331-363. 